\title{
La literatura médica clásica y la arquitectura de las termas medicinales
}

\author{
GLoria Mora
}

La arquitectura termal romana se caracteriza, en términos generales, por su estricta funcionalidad, por la estrecha relación existente entre forma y función. Además de los baños públicos de época republicana e imperial existe otro tipo de termas, las medicinales, que presentan cierta similitud estructural con las anteriores aunque sus objetivos son distintos, ya que se adecúan a los tratamientos médicos prescritos para la curación de enfermedades de todo tipo, especialmente las de origen nervioso, como veremos.

Uno de los aspectos más interesantes del estudio de las termas medicinales es precisamente el análisis de las posibles relaciones existentes entre una forma arquitectónica determinada y una función concreta. En este sentido, nos encontramos con el problema del continuo trasvase de estructuras y sistemas hidroterapéuticos entre las termas a que nos referimos y las de carácter público, trasvase facilitado por las evidentes semejanzas funcionales entre ambas. No obstante, en este breve artículo procuraremos definir en lo posible estas confusiones y determinar en qué medida las necesidades de la medicina experimental contribuyeron a la creación de unas estructuras arquitectónicas especializadas.

En primer lugar, debemos recordar que la palabra griega thermae ( $\theta \in \varrho \mu n ́$, calor), se aplicaba en el mundo romano, en un principio, a aquellos lugares donde existían fuentes naturales de agua caliente con propiedades terapéuticas, así como a las construcciones erigidas para aprovechar estas aguas, en tanto que los términos balneum, balineum o balnea designaban los baños privados y públicos en época republicana, según Varrón (De ling.lat., IX, 68 y VIII, 48). El aprovechamiento de los manantiales termales se desprende de un pasaje de Vitruvio referente a la Campania, región cuya gran actividad volcánica favorecia la prolifera- 
ción de fuentes naturales, y donde se documentan las primeras estructuras de este tipo ya a finales del siglo ill a. C. '?:

«En los montes de la región de Cumas y de Bayas existen cuevas, excavadas para utilizarlas como sudatorios, en las que emerge continuamente del fondo un vapor caliente que perfora, por la vehemencia del fuego, aquella tierra, e, infiltrándose a través de ella, se acumula en aquellos lugares y presta la utilisima ventaja de servir como excelentes sudatorios»? .

Éstas serian, por tanto, las primeras termas medicinales documentadas, asociadas a fuentes naturales de agua caliente, y cuyo fin era conseguir ciertos beneficios para la salud corporal por medio de la sudoración. Hay que tener en cuenta, además, la profunda helenización de la zona de la Campania: como se sabe, la costumbre griega del baño en locales públicos se introdujo en Roma en la segunda mitad del siglo III a. C. ${ }^{3}$, a consecuencia de la intensificación de los contactos con la Campania, tomando ejemplo de los edificios existentes en Capua (los más antiguos, según Tito Livio XXIII, 7, 3, del año 216 a. C.), las Termas Centrales de Cumas y las Stabianas de Pompeya (seguramente el complejo termal más antiguo de Italia, según Nielsen), todos los cuales presentan el esquema típico de los balaneia griegos, añadiendo posteriormente nuevas cámaras y asumiendo dimensiones más amplias ${ }^{4}$.

Pero tras la construcción de los baños de Agripa en Roma entre los años 25 y 19 a. C., que fueron el primer gran complejo termal abierto al público, se empezó a aplicar la denominación de «termas» a los grandes baños públicos, especialmente a los imperiales ${ }^{5}$. Este hecho se debe a la introducción por Agripa en sus baños de una sala especial, llamada laconicum, destinada a los baños de aire caliente, copiada de los baños griegos (la palabra, de hecho, hace referencia a la práctica de este tipo de baño entre los espartanos). El laconicum de las termas de Agripa, en concreto, era una gran sala circular de unos $25 \mathrm{~m}$ de diámetro, de la que sólo se conservan restos relacionados con una reconstrucción posterior de Septimio Severo.

${ }^{1}$ ChIOLINI, P., I caratteri distributivi degli antichi edifici. Milano 1959, págs. 136-137.

${ }^{2}$ Vitr. II, 6.

${ }^{3}$ Plut. Apop. Rom., 10; Justin., XLIV, 2, 6. JohannowskI, W., "La situazione in Campania", en Hellenismus in Mittelitalien, I. Göttingen 1976, pág. 276; BerARD, J., La colonisation grecque de l'italie méridionale. Paris 1957, págs. 37.

${ }^{4}$ Nielsen, I., "Considerazioni sulle prime fasi dell'evoluzione dell'edificio termale romano». Analecta Romana, XIV, 1985, págs. 81-112.

${ }^{5}$ Plin. HN, XXXVI, 104, 121. 
La tipología general de las termas medicinales se caracteriza, precisamente, por la presencia de una estructura circular cubierta por una cúpula, en cuyo centro se abre una claraboya (oculus, lumen) para dejar paso al aire y a la luz, regulada por medio de un escudo o clipeus sujeto con cadenas, con hornacinas o nichos en los muros destinados a contrarrestar los empujes laterales de la cubierta ${ }^{6}$ y con una piscina en el centro llena del agua del manantial, que surge en ese mismo lugar o es llevada hasta alli por medio de canalizaciones. Con frecuencia, la cámara central está rodeada de pequeñas salas con bañeras o piscinas individuales.

Esta sala circular corresponde a la descrita por Vitruvio en el capitulo destinado a la disposición y partes de los baños de sus Diez Libros de Arquitectura:

"Laconicum sudationesque sunt coniungendae tepidario, eaque quam latae fuerint, tantam altitudinem habeant ad imam curvaturam hemisphaerii. Mediumque lumen in hemispherio relinquatur ex eoque clupeum aeneum catenis pendeat, per cuius reductiones et demissiones perficietur sudationis temperatura. ipsumque ad circinum fieri oportere videtur, ut aequaliter a medio flammae vaporisque vis per curvaturae rotundationes pervagetur»?

Como se deduce de este texto y de la nueva sala de las termas de Agripa a que hemos hecho referencia, la estructura del laconicum griego fue adoptada plenamente por los baños públicos (el primer ejemplo de esta adopción sería el sudatorium o laconicum de las termas Stabianas de Pompeya, que data del siglo ॥ a. C. y que fue posteriormente sustituido por un frigidarium). Por otra parte, la dificultad de determinar la función exacta del laconicum ha originado numerosas polémicas al respecto: ¿era una cámara de aire caliente o de vapor? En otro capitulo, el dedicado a la construcción de las palestras ${ }^{8}$ (es decir, los baños propiamente griegos, pues eran non italicae consuetudinis), Vitruvio distingue entre la concamerata sudatio o sala abovedada para sudar y el laconicum, "construído como se indica más arriba», en cuya parte opuesta debia estar el baño de agua caliente (calida lavatio). Es posible que el texto sea confuso y en realidad Vitruvio se esté refiriendo a una gran sala para sudar compuesta por el laconicum y el baño caliente. En cualquier caso, es clara la descripción vitruviana de la estructura y funcionamiento del laconicum, equi-

\footnotetext{
${ }^{6}$ Lugli, G., La tecnica edilizia romana. Roma 1957, pág. 664.

Vitr. $V, 10,5$

${ }^{8}$ Vitr. V, 11, 2.
} 
valente al mugıatńeıov o habitación con aire caliente para sudar de los baños griegos, que Roma introdujo en sus termas ${ }^{9}$ (fig. 1). Los ambientes destinados a los baños de vapor, de los que conocemos la existencia pero no el proceso ni la estructura arquitectónica, llamados assa sudatio o simplemente sudatorium, debian ser similares a la cámara descrita por Vitruvio.

En lo que respecta a las termas medicinales, la estructura y función del laconicum debe ponerse en relación con los tratamientos médicos prescritos para la curación de diversas enfermedades mediante el aprovechamiento de las aguas calientes con propiedades terapéuticas, o bien de la utilización del calor (seco o de vapor) como terapia. Estos tratamientos formaban parte de la práctica de la medicina griega introducida en Roma, en el siglo । a. C., por Asclepiades de Prusa ${ }^{10}$, y básicamente son los siguientes:

a) Curación por inmersión en el agua del manantial ${ }^{11}$.

b) Curación por exposición al vapor de la fuente ${ }^{12}$.

c) Curación por ingestión del agua de la fuente ${ }^{13}$.

El tratamiento por inmersión precisa, lógicamente, una piscina, que por lo general aparece construida sobre el manantial o bien a poca distancia de éste, mientras que el tratamiento por medio del vapor requiere una cámara (laconicum) cubierta con cúpula provista de un óculo regulable, de modo que el vapor pueda ser controlado y no desperdiciado, según las instrucciones dadas por Vitruvio.

Dado que la función y el uso de estas estructuras no cambian, el esquema arquitectónico de las termas medicinales apenas sufre variación

\footnotetext{
${ }^{9}$ Plauto, Stich., 229 habla de «unctiones Graecae sudoratiae»; también Dión Cassio, LIII, 27, 1, y Marcial VI, 42, 16 s.: "ritus si placeant tibi Laconum/contentus potes ariso vapore». Por su parte, Celso, De Med., II, 17 establece que uno de los métodos para lograr el sudor por medio del calor seco es la utilización de los laconici. Sobre la influencia griega en los baños romanos: Ginouves, R., Balaneutiké. Recherches sur le bain dans l'antiquité grecque. París 1962, págs. 147 ss, y B.ALDson, J.P.V.D., Life and Leisure in Ancient Rome. London 1969, pág. 26.

${ }^{10}$ Plin., HN, XXVI, 8 cuenta que Asclepiades fue el primero en emplear el calor proporcionado por los balnea pensilia con fines terápéuticos. También Celso Il, 17, 1-3 explica que Asclepiades recurría a los baños de sudor producidos tanto por el aire seco como por el vapor.

${ }^{11}$ Plin., 11.24 HN, XXXI, 32; Sen., Quaest. nat., III.

${ }^{12}$ Cels., De Med., II, 17.

${ }^{13}$ Una representación de esta práctica aparece en la Pátera de Otañes, donde vemos cómo el agua de la fuente es recogida en un depósito y comercializada en barriles, y cómo le es administrada a un enfermo. También Plin., NH, XXXI, 32.
} 


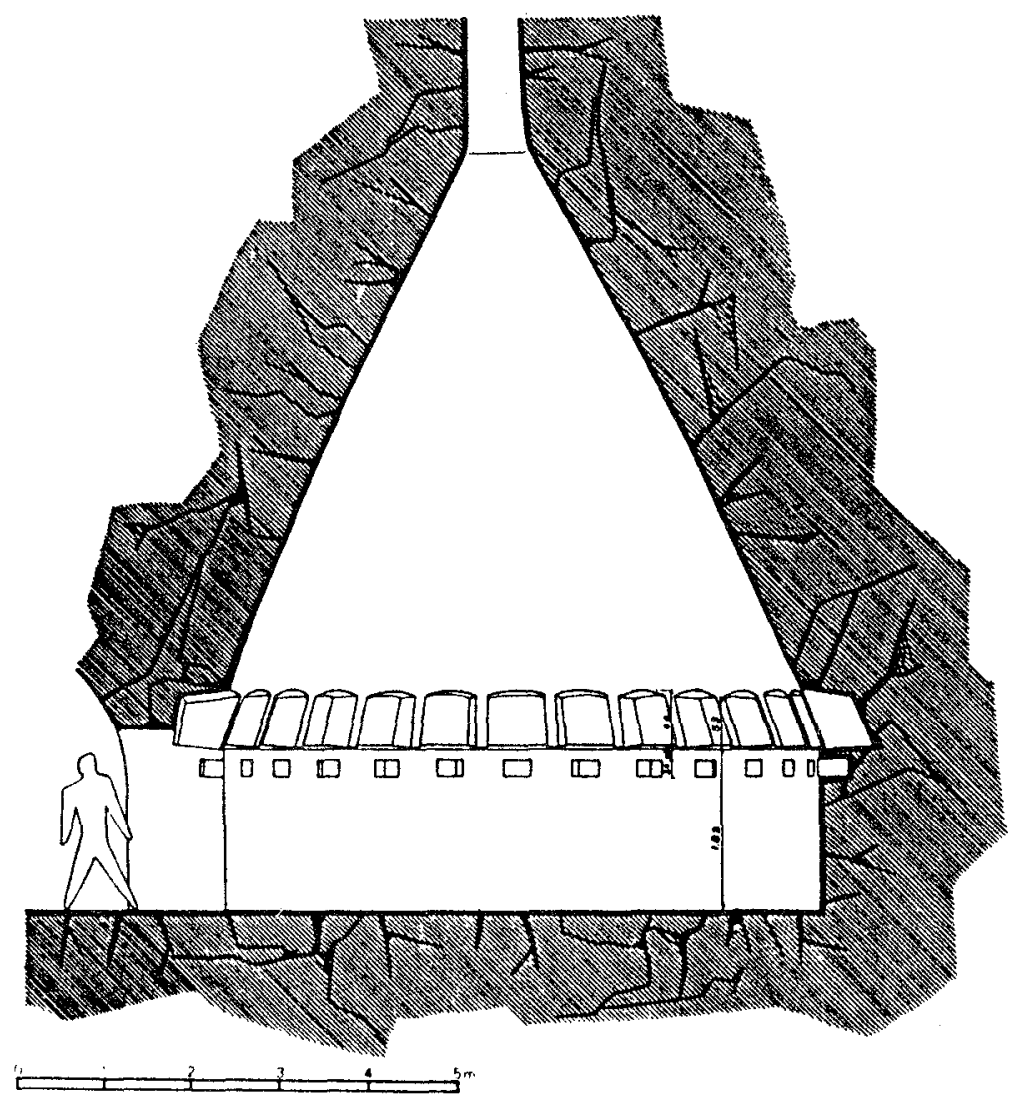

Fig. 1. El Balaneion de El Pireo (según Ginouvès, pl. LVIII).

con el tiempo, manteniéndose igualmente la misma tipología en todos los puntos del Imperio Romano, como demuestran múltiples ejemplos.

El tipo de ambiente característico de las termas medicinales, el laconicum, fue pronto adaptado de forma artificial a los baños públicos, como hemos visto en los casos de Pompeya y las termas de Agripa. Por otra parte, algunas termas de carácter medicinal en su origen, como las de Aquae Sulis, evolucionaron hasta convertirse en grandes complejos termales con la misma funcionalidad que los baños públicos y compaginar ésta con su primitiva dedicación exclusivamente sagrada y salutífera ${ }^{14}$ (fig. 2).

\footnotetext{
${ }_{14}$ Aquae Sulis es una fundación romana del siglo I d. C. sobre un asentamiento anterior
} 
GLORIA MORA

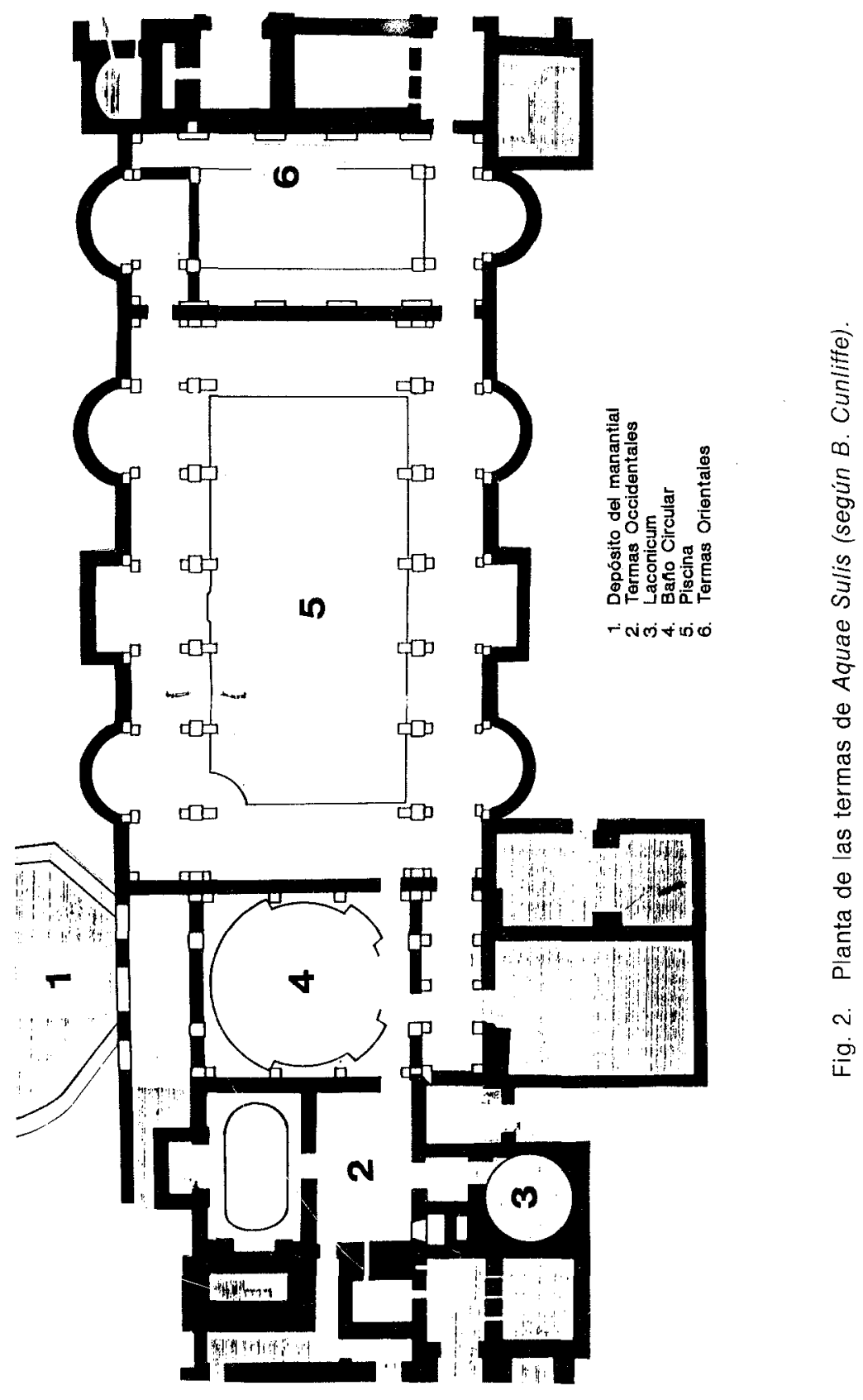


Pero lo más interesante es que junto con la estructura se trasladó también a las termas públicas, a partir de la época de Augusto, la noción de salud ligada a esta práctica del baño de vapor o de aire caliente. La alternancia de éste con el baño de agua fria se convirtió, desde el siglo I d. C., en una práctica perfectamente estudiada y regulada por los tratados de medicina. Celso, Antonio Musa y, sobre todo, Galeno de Pérgamo en el siglo II, establecieron un método hidro-terapéutico basado en la alternancia de calor y frío tras una abundante sudoración, que actuaba eficazmente sobre los problemas circulatorios, favoreciendo la desintoxicación y la reactivación del organismo.

Las fuentes para el estudio de la práctica médica en la antigüedad no son muy abundantes, pero si precisas. Celso, Plinio y Galeno, fundamentalmente, recogen la terapéutica griega, de tipo experimental, que tiene sus raíces en Hipócrates.

Asi, Plinio $(H N, 28,55)$ recomendaba como los mejores remedios que un enfermo podia aplicarse a si mismo el sol y el uso vigoroso de toallas y cepillos para limpiar la piel de toxinas tras un baño de vapor, así como el baño frio tras el caliente. De hecho, después de que Antonio Musa curara a Augusto de una dolencia hepática mediante la prescripción de baños fríos, se puso de moda esta práctica, y en muchos establecimientos termales se añadieron piscinas de agua fría junto al laconicum, como sucedió en las Stabianas de Pompeya y en las termas occidentales de Aquae Sulis, la actual Bath.

Celso, en su tratado De Medicina, II, 17, diserta ampliamente sobre el sudar como terapia médica, y afirma que se puede conseguir por dos medios: mediante el calor seco (el de la arena caliente, de los laconica y de los hornos, y el de «algunos lugares naturales donde el vapor caliente exhalado del suelo se encierra en un edificio, como en las grutas de Baias"), y mediante el baño caliente. Estos tratamientos son útiles para expulsar los humores nocivos del interior del cuerpo humano y dispersarlos, y también para curar enfermedades nerviosas. El baño de vapor, en concreto, sirve para eliminar la opresión de la región del corazón y de la garganta, así como la que daña algún miembro, ya que resulta beneficioso para los nervios contraídos, que son la causa de estos males.

en torno a un manantial de aguas medicinales (indicadas en el tratamiento del reuma, hipertensión y desórdenes metabólicos) asociado al dios Sul, identificado posteriormente con Minerva Sulis. A ambos lados del gran depósito que recogia el agua del manantial se construyeron en épocas distintas dos complejos termales con las estancias propias de los baños públicos: of. CUNLIFFE, B., Roman Bath discovered. London 1971. 
Por lo que se refiere a las aguas minero-medicinales, Plinio dedica el capitulo 32 del libro XXXI de su Naturalis Historia a las aguas termales y sus aplicaciones en medicina, como así Vitruvio VIII, 3, 4-5, reflejando los métodos de la terapéutica griega practicados en la época, y distinguiendo claramente el uso de los distintos tipos de agua:

«El agua sulfurosa es buena para los nervios, la aluminosa para las parálisis y astenias del mismo tipo, la bituminosa o nitrosa para bebidas y purgas. Muchas personas se jactan de soportar durante largo tiempo el calor de las fuentes termales, lo que es muy nefasto: en efecto, su uso no debe ser apenas más prolongado que el del baño, hay que seguirlo de abluciones de agua fria y no marcharse sin frotarse con aceite, práctica que, a ojos del público, es perjudicial...».

Advierte también del peligro de beber demasiada agua, porque produce hinchazón (también Vitruvio, VIII, 3, 5). Alude a la utilidad de los baños de barro procedente de las fuentes termales, pero aconseja dejar secar al sol la capa de barro para que sea efectiva. Diserta a continuación sobre aquellas aguas consideradas medicinales pero que no tienen nada de tales, como las de Segesta, en Sicilia, Larissa en la Tróade, Magnesia, Melos y Lipara, y también sobre los beneficios de la hidroterapia marina.

Acerca de la natación, otro de los métodos terapéuticos empleados por los médicos griegos, Plinio la rechaza en el caso de los que sufren cefaleas, debido a la diferencia de temperatura que se establece entre la cabeza y el resto del cuerpo y que puede agravar el mal ${ }^{15}$.

Los métodos terapéuticos empleados en las termas de aguas mineromedicinales, y relacionados con los distintos usos del agua, pasaron pronto a la práctica cotidiana y fueron empleados indistintamente en las termas de carácter urbano. Con respecto a los baños, Galeno de Pérgamo, médico de Marco Aurelio, refundió los consejos emitidos por diversos autores en el siglo । d. C., estableciendo cuatro momentos esenciales:

1. ${ }^{\circ}$ Sudar mediante el ejercicio o la permanencia en el laconicum, porque el sudor ayuda a expulsar los malos humores y a limpiar la piel.

2. Tomar un baño caliente y frotar la piel con strigiles para eliminar la suciedad y las toxinas.

$3^{\circ}$ Continuar con un baño frío por inmersión, para vigorizar el cuerpo y activar la circulación.

${ }^{15}$ También Cael. Aurel., Chron., 1, 42. 
4. ${ }^{\circ}$ Finalmente, darse masajes y unciones con aceite y ungüentos, para defender el cuerpo de la temperatura exterior ${ }^{16}$.

Los métodos terapéuticos griegos recogidos por Galeno en el siglo ॥ siguieron aplicándose durante toda la época imperial, hasta el punto de ser compilados todavía en el siglo iv por Oribasio, médico del emperador Juliano, en su Synopsis. En un pasaje del tratado dedicado a los baños, Oribasio formula prescripciones muy precisas sobre la duración de los baños y las curas con aguas minerales: si el tratamiento va a durar tres semanas, recomienda empezar por bañarse durante media hora y aumentar la duración del baño hasta llegar a las dos horas al séptimo día, continuar asi durante la segunda semana, y a partir de la tercera disminuir el tiempo en la misma proporción que al comienzo.

Por lo que se refiere a España, existen numerosos ejemplos de termas medicinales, muchas de las cuales han venido funcionando desde época romana (e incluso prerromana) hasta hoy. En un trabajo anterior ${ }^{17}$ hemos contabilizado al menos una veintena de balnearios actuales construidos sobre antiguas termas aprovechando las estructuras supervivientes de éstas, de los cuales el más conocido y probablemente el mejor conservado es el de Alange, en Badajoz. Las termas de Alange, bien estudiadas por José María Álvarez ${ }^{18}$, presentan la estructura típica de laconicum descrita por Vitruvio, aunque sus aguas minero medicinales no podian por sí solas, dada su baja temperatura $\left(27^{\circ}\right)$ provocar el vapor necesario para la curación por este método (fig. 3). Aquí, pues, tendríamos un ejemplo claro de la aplicación de una estructura establecida por los textos clásicos de arquitectura en un edificio que no reúne las condiciones adecuadas para la producción de calor de modo natural. Este hecho debe considerarse como un ejemplo más del reciproco trasvase formal y funcional que hemos observado anteriormente, con respecto al caso de Aquae Sulis, entre las termas de carácter puramente medicinal y los baños públicos.

${ }^{16}$ Galen. Meth.med. X, 10 y XI, 10. También Cels., II, 17; III, 21; I, 4; Plin., HN, XXXVIII, 55; Plin., Ep., 9, 36, 3.

17 Mona, G., "Las termas romanas en Hispania», AEspA, 54, 1981, págs. 37-89. 290.

18 Álvarez Martínez, J. M., "Las termas romanas de Alange", Habis, 3, 1972, págs. 267- 
GLORIA MORA
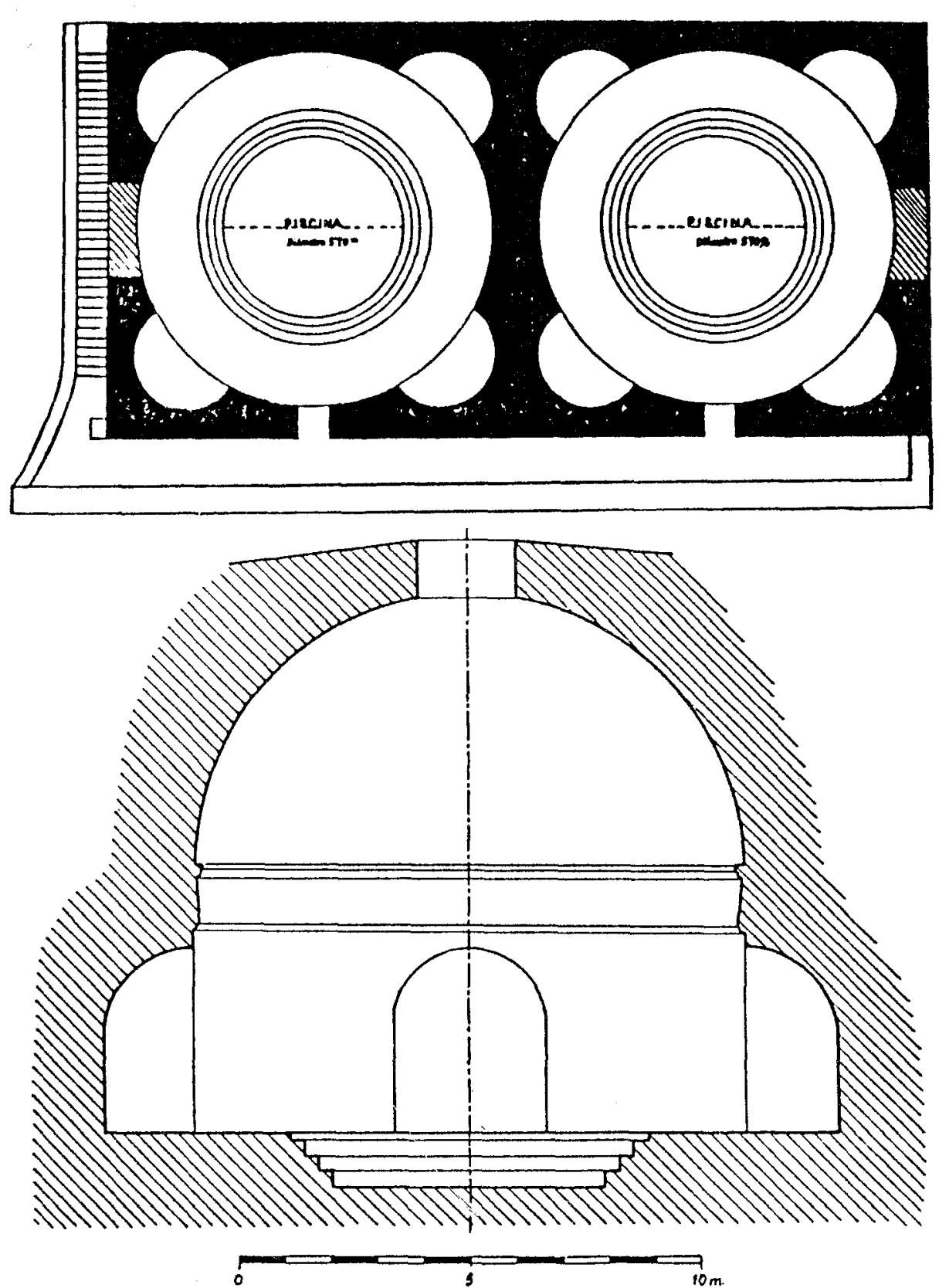

Fig. 3. Planta y sección de las termas de Alange (según J. M. Álvarez). 


\section{RESUMEN}

En este articulo se analiza la relación entre la arquitectura de las termas medicinales, caracterizada por la presencia de la cámara denominada laconicum, y los tratamiemtos basados en las virtudes terapéuticas de las aguas minero-medicinales prescritos por la medicina experimental de origen griego. Para ello se han estudiado los textos clásicos referentes al tema, tanto el Tratado de Arquitectura de Vitruvio como los escritos sobre medicina de Celso, Galeno, Plinio y otros autores.

\section{ABSTRACT}

This article analyses the relationship between the architecture of medicinal spas, such as those with a laconicum, and treatments based on the therapeutical virtues of medicinal mineral waters as prescribed by Greek doctors. The analysis is based on the study of classical texts on the subject, both the ten books on architecture by Vitruvius and the medicinal treatises of Celsus, Galenus, Pliny and other authors. 